\title{
SORTS AND SOURCES OF STRESS IN PAKISTAN; A COMPREHENSIVE OUTLOOK
}

\author{
Sadaf Ahmed ${ }^{1 \& 3} \&$ Shamoon Noushad ${ }^{1 \& 2}$ \\ 1. Advance educational institute \& research Pakistan (AEIRC) \\ 2. Institute of basic medical sciences, /Dow University of health science, Pakistan \\ 3. Department of physiology, university of Karachi
}

Corresponding author: shamoon@aeirc-edu.com

\begin{abstract}
Objective: to evaluate Psycho-physiological cause and developing burden that account for stress with variability in terms of duration, intensity and healthy or unhealthy effects on physical and emotional wellbeing. Background: In Pakistan the condition of physiological and psychosocial health is becoming weaker with time due to occurrence of non peaceful and haphazard life resulted in constant stress. Method:. There are various ways of analyzing stress from observing symptoms, change in behaviors. These can be lead to body either towards strength, improved body reactions and better performance as psycho-physiological being. We have highlighted here the emerging stressors in Pakistan and their effects on body \& mind. Result: The psycho-physiological conditions result of experiencing changes in homeostatic balance, these imbalances either lead to positive or negative impact on health status of well being and trigger three main pathways physiologically Conclusion: In this editorial the predictable indicators of stress response and there positive and negative influences are summarized with respect to emerging and existing stressors known to effect this set of population. Application: This is a theoretical evidenced based psychoanalysis will be leading towards designing an inventory specifically based on recent stressors acting on human body and types of induced stress.
\end{abstract}

\section{KEY WORDS}

Stress, Psychophysiology, Pakistan, Stressors, psychological, physiological

\section{INTRODUCTION}

The status of well being in Pakistan is currently weak (WHO, 2006) and is becoming weaker with every passing day as continuous occurrence of roller-coaster political history which has resulted in confusing the meaning of the state as understood in Pakistan (Easterly, W. 2001). With constant stress, terrorist attacks, absence of social security and poverty, Pakistan is fast becoming a "psychological pressure cooker"( Jamali, A. R ,2000), there is an epidemic of Post-Traumatic Stress Disorder (PTSD) in Pakistan (Hashmi, S, 2011) due to anxiety induced by lack of basic necessities, religious extremists, social adversity, low income, illiteracy (Jamal, A. 2006).. Most people in Pakistan are living with a continued sense of helplessness (Karim, S.,2004), and rather than dealing with it they are accepting it, the life difficulty schedule is much more adverse with psycho- social efforts in Pakistan(Michael, S. 2007). It is well understood though that when your physical well-being is not guaranteed and you don't have enough to feed your children your mental health is not a priority but the pressures in directly or directly affecting majority of the population psycho-physiologically either as chronic or acute stressors(Sonnentag, S., \& Fritz, C. 2006). The persistence of the lackings and hardships and the worsening of conditions augmented the intensity of hits on mind and body. This is causing a sort of illness both mental and physical without coping with it or in other words, people have become very rigid, inflexible in their thinking, and confused about religion. Things are going badly wrong. People are unable to rationally analyze the situation and are becoming angrier. The increase prevalence of health maladies like insomnia, hypersomnia, hypertension, diabetes, angina, ulcers, reproductive \& sexual disabilities, cognitive impairments, suicide, irritability, anxiety, tiredness, low self esteem has been continuously increasing in our population due to nonstop burden of stress with its unique classical and contemporary varieties.

\section{Psycho-physiology of stress}

The psycho-physiological conditions/adaptations of an individual body as result of experiencing changes in homeostatic balance, these imbalances either lead to positive or negative impact on health status of well being. According to a psycho physiologists : "stress is an outcome of diverse dealings (Wolf, T. M. 1994)with influencing factors like pleasure or pain(Grant, J 2006) that could be tolerable or intolerable and require additional resources from body that imbalances homeostasis leads to initiation or stimulation of certain physiological responses of the internal environment, in a positive or negative mode (Watson, D. 1988)." On the whole pattern of physiological, emotional, cognitive and behavioral response to a number of enormously challenging facets can lead towards anomalous suppressed states that can be termed as stress.( Fonagy, P. 2001 ) The established psychological representation of stress and disease suggests that we assess the level to which conditions in our surroundings are ominous; we react to assess pressure with negative emotion leads to physiological alteration that may influence disease commencement and sequences. There is a sequential phenomenon involved from individual single cell level to multicelluler disrupted functions vary from organism to organism.( Nicholson, J. K., 2004) The overall mechanisms is a whole set of events and biological changes with respect to have the ability to respond to stressors(Anisman, H 1999), that involve a multifaceted set of connections from sensing and signal transduction to adaptations and proliferation as well as gene expression programming that could be resulted in variations of metabolic activities, physical characteristics or survival of a cell also be referred as cellular stress(Ibegbu, A. 2009). There are countless sources of stress known as stressors (Lazarus, R. S 1986) from any frightening or complicated incident in life to manage to impending surplus pressures(Hoehn, T., 1997). It is important to bear in mind that stress is an individualistic, subjective experience and therefore what one person finds stressful another may not (Wegner, D. M., 1987). Stressors can be broken down roughly into either external or internal (or a mixture of both) (Levins, R. 1998).

\section{Psychophysiological phenomenon of Stress}

Physiological influences of psychological stress trigger 3 main pathways related to stress are nervous, endocrine and immune system. (Chrousos, G. P. 2009) Neuroendocrine stress response is 
obvious after the experience to broad range of stressors resulting in positive or negative stimulus to many factors in the brain, endocrine glands and other organs(Goldstein, D. S 2007). This numeral of factors is still under research, but according to classic stress perception Whenever there is an interruption in normal environment the responsive HPA communicates through $\mathrm{CRH}$ and $\mathrm{ACTH}$ and releases Stress Hormones commonly known as Glucocorticoids \& catecholeaminess influencing a body to produce Fight or flight response, changes in heart rate and blood pressure mainly (Black, P. H. 1995).mExperiencing stressors results in a sequence of synchronized responses not only to improve the prospects of endurance but also to alleviate sufferings. This can be majorly done by alterations in behavior, autonomic function and neuro endocrine secretions, longer-lasting activation of the hpa-axis is associated with activation of the renin-angiotensin system initially(Kaye, J. M 2005), within seconds releases augmented secretions of catecholamines(Urhausen, A. 1995). CRF and vasopressin from parvicellular neurons into the portal circulation and increased secretion of oxytocin from the neural lobe of the pituitary, and 5-10 s later (Lightman, S. L.,1988), the secretion of pituitary ACTH that also involves, some seconds later, a decreased secretion of pituitary gonadotropins and increased secretion of prolactin and growth hormone from the anterior pituitary, increased secretion of renin from the kidneys and the pancreatic secretion of glucagon one minutes later(Carrasco, G. A.,2003), there is an increase in the plasma levels of glucocorticoids and an inhibition of the secretion of gonadal steroids (Tilbrook, A. J 2000). However, the glucocorticoid peak appears between $30 \mathrm{~min}$ and $1 \mathrm{~h}$ after the onset of the Several neuropeptides and neurotransmitters other than CRF also regulate the stress response in a coordinate way(Carrasco, G. A.,2003), each following a determined time course and specificity for a determined stressor. This whole phenomena leads to recruitment of energy to keep brain and muscle functioning; sharpening and focused attention on the perceived threat; increased cerebral perfusion rates and local cerebral glucose utilization(Kaye, J. M 2005); improved cardiovascular output and respiration, redistribution of blood streaming, increasing substrate and energy delivery to the brain and muscles; modulation of immune utility; reduced reproductive function and sexual deeds; decline in desire for food (Borer, K. T. 2003). . In the specialized situation of fluid loss due to hemorrhage, responses also include water retention through both renal and vascular mechanisms (Sapolsky, R. M., 2000). There are various ways of analyzing stress from observing symptoms, change in behaviors other screening marker as development of new techniques made possible to analyze small amounts of biologically active substances in the blood and tissues and the information gathered enabled enlargement and reevaluation of the stress theory. One of the questions repeatedly discussed is the specificity of the stress response. But the criteria and evaluation can be confusing as these strains leads body towards numerous responses that can be positive or negative, acute or chronic to body.

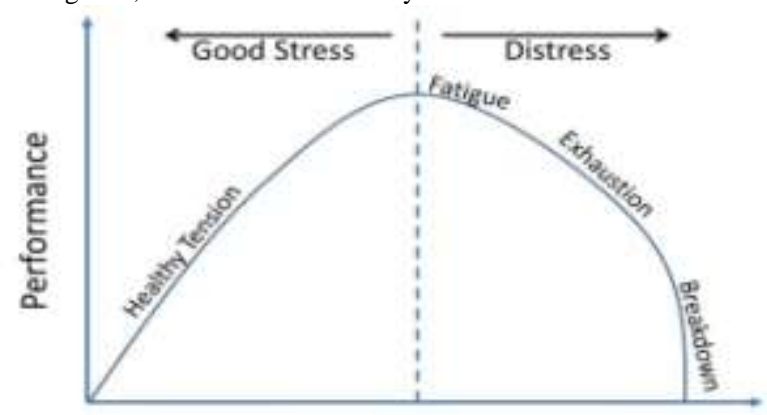

Stress Level

\section{CATEGORIES OF STRESS}

Acute positive stress (arise fast - eustress)

Good (positive) kind of stress that provides immediate strength as winning a game or race, be with partner, child birth etc. facilitate individuals improved performance to face challenges, situations that need an extra push and sometimes necessary for survival and prosperity in many parts of life. it physiologically occurs for a short period of time and it is still stress and it is not good to be so strong or act too often. It is usually immediate and intense reaction of body and mind in small doses and populate as pleasant, cheerful, thrilling, exciting stress or feelings.

\section{Acute negative stress (arise fast- distress)}

Devoid of control, the stress becomes bad stress or distress that not only weakness body or mind but also make us vulnerable to illness. e.g sudden death of family member, road accident, rejection from a job interview etc. indicated mostly too much by mental health problems, physical difficulties, tension headaches, upset stomach, exhaustion. these symptoms are recognized by most people and it is highly treatable and manageable stress.

\section{Chronic positive stress: (long term- good stress)}

Can turn into bad stress, and vice-versa. Our hormones change, but there is no threat or fear. it keeps us feeling alive and excited about life. e.g. relation with newborn baby, leader in a society/group. Having yoga exercise regularly etc. but Long-term exposure to even positive stress can lead exhaustion of nearly every system in your body. It can raise blood pressure, suppress the immune system, increase the risk of heart attack and stroke, contribute to infertility, and speed up the aging process. Long-term stress can even rewire the brain, leaving you more vulnerable to anxiety and depression as prolong exposure to excitements, challenges and competitions etc.

\section{Chronic negative stress}

when a long term stressor is present around for a phase or prolong period of life results in chronic stress that could be dangerous in many ways first, because of people get used to it and ignore this stress harming their mental and physical health secondly persistent of pressures can lead to even more retarded health conditions as diabetes, hypertension. This is even more disastrous condition as individuals are recognized as patients, get trapped in an unhappiness and never-ending "troubles", cannot find a way out of a desperate situations and continuously feeling sick.

\section{Physical Stress}

Any exerted force that is characterized by vigorous bodily activity can lead to altered physiological responses is counted as physical stress such as aerobics, work outs, injury or aches. It may also count on physical traumas as cuts, burns, sprains, broken bones, surgery, etc. In other words physical stress is bodily response to substantial pressures such as exertion, noise, illness or exercises.

Types: Positive \& negative

Signs and symptoms: Breathlessness, Churning stomach, Diarrhoea/Constipation, Dizziness, Dry mouth,Excess perspiration, Fatigue, Headaches, Hyperventilating, Increased colds/flu, Increased heart rate, Indigestion, Impotence, Nausea, Palpitations, Pre Menstrual Syndrome, Sense of heart pounding, Shallow breathing, Sleeping problems, Tension headaches, Tingling in hands/legs, Tremor in hands/legs, Weight loss/gain. 


\section{Traumatic stress}

Exposure to any trauma such as criminal victimization, natural disaster, war and terrorism, witnessing or confronting such situations or itself experiencing them as actual or threatened death (or serious injury) to self or others can dwell a person into traumatic stress. The feelings of intense fear, helplessness, or shock may occur that can cause an acute stress disorder (ASD) or post-traumatic stress disorder (PTSD). The acute symptoms might be recovered in few days to week but the later one can be for a longer period of time and must be treated psychologically more.

Types: Negative

Signs and symptoms: feeling of traumatic events happen again, mental and physical discomfort when reminded of the traumatic event (e.g.on the anniversary of the traumatic event), No expression of emotions, periodic painful dreams, Bad memories of the traumatic event, easily anxious, Avoid discussion of that event, reduced interest or participation in significant activities, disconnected feeling from others or reality,

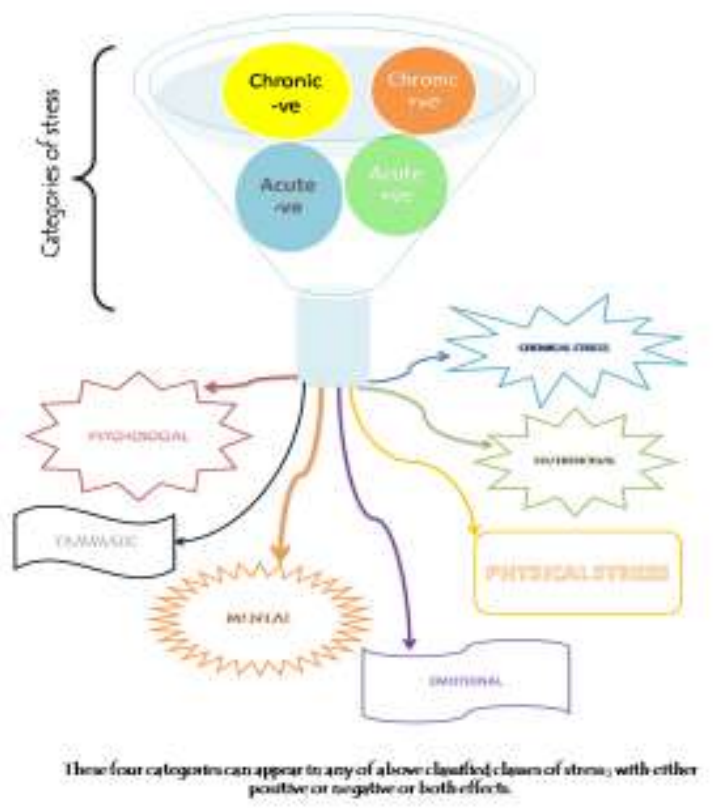

\section{Nutritional stress}

Every chronic state creates particular requirement for certain types of nutrients and antioxidants. the longer the deficiency of nutrients, the greater will be the damage that can be termed as nutritional stress. Eating a well balanced diet and supplementing creates a baseline of health. Dealing with excessive levels of stress hormone is the key to lowering the body's damage due to stress and eases the burden on your natural system of detoxification along with Irregular eating behavior that triggers biological system and body uses energy at a faster rate lead to malfunctions both physically and mentally.

\section{Types : Negative}

\section{Signs and symptoms:}

Pallor, decrease frequency of urination , increase blood pressure, Sleep deprivation, Fatigue, Mental fog, Irritability, Increase/decrease blood glucose, decrease wbc, abnormal thirst, Sugar, Carbs \& Caffeine Craving, gain or lose weight, indigestion, abnormal bowel movements, Pass a hard stool fewer than three times a week, Strain frequently during bowel movements, A sense of incomplete emptying after a bowel movement, Have abdominal bloating or discomfort, Pain in pelvis during passage of stools.

\section{Emotional stress}

Any sort of emotional annoyance/interruption can be counted as emotional stress whether acute or chronic. It can lead to significant chronic health challenges because body cannot defend itself against the damage that emotional stress quietly creates over time and suffers with heavy physiological price for every feeling of anxiousness, tenseness, frustration, anger or even excitement at toxic level. These all emotions serve important purposes when they first arise. The danger is in experiencing these emotions on a chronic basis.

Types: Positive \& negative

Sign and symptoms: Moodiness, irritability or short temper, agitation, inability to relax, feeling overwhelmed Sense of loneliness and isolation, depression or general unhappiness, Anxiety, Worrying, Depression, Sadness, Feeling insecure, Lack of focus, Burnout, Forgetfulness, Crying spells ,Relationship conflicts, Abnormal Laughter.

\section{Mental stress}

Kind of mental arousal or pressure that can be evoked by a number of mental tasks-e.g., mental arithmetic, maze solving or a mentally troubled condition is called the mental stress . Major life events as changes in work, financial problems, studies load, children and family problems are few of these.

Mental stress is seen to affect parts of the brain where memories, perception, thoughts and decision making are processed through and stored along with vital motor activities.

Types: Positive \& negative

Sign and symptoms: memory problems, inability to concentrate, poor judgment, seeing only the negative, anxious or racing thoughts, constant worrying, Relaxation, Low Self-Esteem, Panic Attacks, Insomnia, Hyperactivity, Obsessive Thoughts, Repetitive thinking, Poor Judgment, Disorientation, Detachment, Nightmares, Difficulty in Thinking.

\section{Psychosocial stress}

Experiencing pressure or demands that originate from any type of interaction with people, society or perceived requirement of social lives/belongings (real or even imagined) can challenge your social and psychological well being. This is combinedly known as psychosocial stress which is not caused by single event but by ongoing problem in personal and social interactions as a part of daily routine and behaviors.

Types: Positive \& negative

Sign and symptoms: Absenteeism/presenteeism, Accidents, Inability to delegate, Increased sick days, Inefficiency, Persistent lateness, Procrastination, Poor decision making, Poor interaction with colleagues, Reduced work performance, Resentment/anger/irritability, Aggression, Agitation, Avoidance behavior, Crying, Decreased/increased sexuality, Difficulty relaxing, Difficulty with relationships, Eating fast, Eating too much/too little, Gambling, Hostile behavior, Impatience, Increased alcohol, Increased caffeine, Increased smoking, Losing temper, Making mistakes, Nail biting, Outbursts of anger, Poor eye contact, Poorer personal hygiene, Poor time management, Restlessness, Risk taking, Substance, abuse, Talking fast, Walking fast, Withdrawal from relationships, Withdrawal from activities 


\section{Chemical stress}

The endogenous or exogenous have the ability to reduces bodily ability to cope with environmental changes and cause damage or irritation to vital organs or sensitive tissues this sort of forces are responsible to induce chemical stress which result in a reflex to the spine creating spinal imbalances and subluxations or as high sugar and fat content of fast food, combined with lack of exercise, creates a chain of events that put the body into chemical stress that in turn creates the hormones that create the belly fat.

\section{Types: Negative}

Sign and symptoms: use of caffeinated drinks, like cozy rooms (deoxygenated places), take less than four glass of water, use of medicines daily, body temperature, feel dehydrated, Shortness of breath, Chest pain, Irregular heartbeat, Heart attack (rare), Hypersensitivity to food additives or other, Insensitivity to heat/cold

\section{CONCLUSION AND FUTURE PROSPECTS}

The past 2 decades have seen a dramatic increase in factors affecting physical and mental health consequences of traumas, negative events, and chronic strains. Psychosocial burden also account for stress with variability in terms of gender, race, ethnic, age, marital status, and socioeconomic status differences in physical and emotional wellbeing. These findings point insistently to the origins of spoiled health a way to satisfactory health inequalities in the comprehensive long run. In their personal family, and work lives individuals and populace as a whole are coping and struggling with key events or unceasing strains that in many circumstances and stronger beings respond for the acceptance e.g. The intensity of anxiety, fierceness is increasing yearly as the situations of load shedding are getting worse. The population is continuously losing their energies and time management become impossible for them (Sadaf, 2011). On the other hand as it is suggested that cognitive impairment is related to lifestyle, educational level, depressive and stressful life. Low socio-economical status, low educational level and stress and depression cause negative effect on memory and cogitative function (Sadaf, 2012). As our teenagers are becoming depressed due to day to day issues. Social and psychological pressures amplified in our society it is making conditions worse in terms of substance abuse that can make lifestyle either life looks better (Faizan \& Sadaf, 2013) or addiction for feling pleasure and to cope out stress that could be a random factor to satisfy brain hence body(Azher et al 2012). Nicotine smokers mainly in our study were poorly educated in terms of health consequences and risks of many diseases that could be vulnerable as they continue to smoke moreover they also do not benefited by the non motivating anti tobacco campaigns as their urge to smoke is much stronger. These negative stressors can be encountered by inducing healthy and controlled eustress as engaging one's self in physical activity or work outs that not only have a profound effect on physiological parameters to raise health standard (Shamoon, 2012) but also to minimize mental shutdown (Sadaf, 2011).

\section{KEY POINTS}

- $\quad$ Psychosocial burden account for stress with variability in terms of gender, race, ethnic, age, marital status, and socioeconomic status differences in physical and emotional well-being.

- The criteria and evaluation can be confusing as these strains leads body towards numerous responses that can be positive or negative, acute or chronic to body.

- The predictable indicators of stress response and there positive and negative influences are summarized with respect to emerging and existing stressors known to effect this set of population.

\section{ACKNOWLEDGEMENT}

We would like to express kind gratitude to Mr. Syed Zain Azher, Mr. Shaikh Mohammad Tahir, Ms. Saima Khan \& Ms. Maria Altaf who gave their valuable input \& suggestions in the possibility of compiling this article and information without them this meticulous evaluation will not be possible.

\section{REFERENCES}

- Anisman, H., \& Merali, Z. (1999). Understanding stress: characteristics and caveats. Alcohol Research and Health, 23(4), 241-249.

- Black, P. H. (1995). Psychoneuroimmunology: brain and immunity. Scientific American Science and Medicine, 2, 16-27.

- $\quad$ Borer, K. T. (2003). Exercise endocrinology. Human Kinetics 1.

- Carrasco, G. A., Van de Kar, L. D., \& Carrasco, G. A. (2003). Neuroendocrine pharmacology of stress. European journal of pharmacology, 463(1-3), 235-272.

- Chrousos, G. P. (2009). Stress and disorders of the stress system. Nature Reviews Endocrinology, 5(7), 374-381.

- Country Cooperation Strategy for WHO

- $\quad$ and Pakistan 2005-2009 World Health Organization Regional Office for the Eastern Mediterranean Cairo, 2006

- Easterly, W. (2001). The political economy of growth without development: A case study of Pakistan. Paper for the Analytical Narratives of Growth Project, Kennedy School of Government, Harvard University.

- $\quad$ Faizan Mirza \& Sadaf Ahmed. 'Psychological effects of using power-enhancing drugs on gym-exercising individuals.' Vol 4, Issue 4, 20131565-1568

- Fonagy, P. (2001). Attachment theory and psychoanalysis. Other Press

- Goldstein, D. S., \& Kopin, I. J. (2007). Evolution of concepts of stress. Stress: The International Journal on the Biology of Stress, 10(2), 109-120

- Grant, J. E., Brewer, J. A., \& Potenza, M. N. (2006). The Neurobiology of Substance and Behavioral Addictions (2006). CNS spectr, 11(12), 924-930.

- Hashmi, S., Petraro, P., Rizzo, T., Nawaz, H., Choudhary, R., Tessier-Sherman, B., ... \& Nawaz, H. (2011). Symptoms of anxiety, depression, and posttraumatic stress among survivors of the 2005 Pakistani earthquake. Disaster medicine and public health preparedness, 5(4), 293.

- Hoehn, T., Braune, S., Scheibe, G., \& Albus, M. (1997). Physiological, biochemical and subjective parameters in anxiety patients with panic disorder during stress exposure as compared with healthy controls. European archives of psychiatry and clinical neuroscience, 247(5), 264-274

- Ibegbu, A. (2009). The effects of Hypoxia on neuronal cell signalling (Doctoral dissertation, Queen Margaret University).

- Jamal, A. (2006). Gender, Citizenship, and the Nation-State in Pakistan: Willful Daughters or Free Citizens?. Signs, 31(2), 283-304.

- Jamali, A. R., Ghulamullah, S., Qureshi, I., \& Mehboob, G. (2000). Human cost of political violence. JOURNALPAKISTAN MEDICAL ASSOCIATION, 50(1), 25-28.

- Karim, S., Saeed, K., Rana, M. H., Mubbashar, M. H., \& Jenkins, R. (2004). Pakistan mental health country profile. International Review of Psychiatry, 16(1-2), 83-92. 
- $\quad$ Kaye, J. M., \& Lightman, S. L. (2005). Psychological stress and endocrine axes.Human psychoneuroimmunology, 25-52.

- Lazarus, R. S., \& Folkman, S. (1986). Cognitive theories of stress and the issue of circularity. In Dynamics of stress (pp. 63-80). Springer US

- Levins, R. (1998). The internal and external in explanatory theories. Science as Culture, 7(4), 557-582.

- Lightman, S. L., \& Young, W. S. (1988). Corticotrophinreleasing factor, vasopressin and pro-opiomelanocortin mRNA responses to stress and opiates in the rat. The Journal of physiology, 403(1), 511-523.

- $\quad$ Michael, S. (2007). Terrorism a Socio-Economic and Political Phenomenon with Special Reference to Pakistan. Journal of Management and Social Sciences,3(1), 35-46.

- Nicholson, J. K., Holmes, E., Lindon, J. C., \& Wilson, I. D. (2004). The challenges of modeling mammalian biocomplexity. Nature biotechnology, 22(10), 1268-1274.

- Sadaf A. Shamoon N. \& Tooba S.\& Hiba J. The influence of exercise on physical and mental fitness of exercising young males. Int. J.biol.biotech., 8(3): 445-448, 2011.

- Sadaf Ahmed, Madiha Shaukat, Aleena Hasni \& Shamoon Noushad. Psycho -social stress as a risk factor for cognitive decline in houswives. Indian journal of health and wellbeing, 2011, 2(5) 935-937.

- $\quad$ Sadaf, A., Shamoon, N. \& Faiza, A. Electricity Load Shedding As A Major Cause Of Fierceness, Anxiety And Impaired Intellectual Functions. Indian Journal of Health and Wellbeing, 2011, 2(5) 935-937.

- $\quad$ Sapolsky, R. M., Romero, L. M., \& Munck, A. U. (2000). How do glucocorticoids influence stress responses? Integrating permissive, suppressive, stimulatory, and preparative actions. Endocrine reviews, 21(1), 55-89.
- $\quad$ Shamoon, N. \& Sadaf, A. Effect of exercise on hematological paramaters; a study on trained vs un-trained male subjects. Pak. j. life soc. Sci. (2012), 10(1): 18-21

- $\quad$ Sonnentag, S., \& Fritz, C. (2006). Endocrinological processes associated with job stress: Catecholamine and cortisol responses to acute and chronic stressors. Research in occupational stress and well-being, 5, 1-59.

- Tilbrook, A. J., Turner, A. I., \& Clarke, I. J. (2000). Effects of stress on reproduction in non-rodent mammals: the role of glucocorticoids and sex differences. Reviews of reproduction, 5(2), 105-113.

- Urhausen, A., Gabriel, H., \& Kindermann, W. (1995). Blood hormones as markers of training stress and overtraining. Sports medicine (Auckland, NZ), 20(4), 251-276.

- Watson, D. (1988). Intraindividual and interindividual analyses of positive and negative affect: their relation to health complaints, perceived stress, and daily activities. Journal of personality and social psychology, 54(6), 1020.

- Wegner, D. M., Schneider, D. J., Carter, S. R., \& White, T. L. (1987). Paradoxical effects of thought suppression. Journal of personality and social psychology,53(1), 5-13.

- Wolf, T. M. (1994). Stress, coping and health: enhancing well-being during medical school. Medical Education, 28(1), 817 .

- Zain Azher, Sadaf Ahmed, Shamoon Noushad, Zainab Qudsia \& Azam Yaseen. The major apprehensions in young chronic cigarette smokers to quit smoking. Funast $j$ biol. (2012), fuuast j. Biol., 2(2): 59- 